\title{
BIOMARKERS OF OVARIAN FUNCTION IN GIRLS AND WOMEN WITH CLASSIC GALACTOSEMIA
}

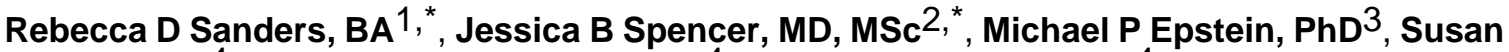 \\ V Pollak, BA ${ }^{4}$, Pratibhasri A Vardhana, MD ${ }^{4}$, Joyce $\mathrm{W}^{2}$ Lustbader, MD $^{4}$, and Judith $\mathrm{L}$ \\ Fridovich-Keil, PhD $^{3}$ \\ ${ }^{1}$ Graduate Program in Biochemistry, Cell, and Developmental Biology, Emory University School of \\ Medicine, Atlanta, GA, 30322 USA \\ ${ }^{2}$ Dept. of Gynecology and Obstetrics, Emory University School of Medicine, Atlanta, GA, 30308 USA \\ ${ }^{3}$ Dept. of Human Genetics, Emory University School of Medicine, Atlanta, GA, 30322 USA \\ ${ }^{4}$ Dept. of Obstetrics and Gynecology, Columbia University College of Physicians and Surgeons, \\ New York, NY, 10032 USA.
}

\section{Abstract \\ Study objective-To determine whether premature ovarian insufficiency (POI) associated with classic galactosemia results from a true deficiency of ovarian function or from aberrant follicle stimulating hormone (FSH).}

Design-Cross-sectional study

Setting-University research laboratory

Patients or other participants-Study subjects included 35 girls and women with galactosemia and 43 control girls and women between the ages of $<1$ yrs to $51 \mathrm{yrs}$.

Interventions-Blood sampling and medical and reproductive histories were obtained.

Main outcome measures-We determined FSH and anti-Müllerian hormone (AMH) levels in subjects with and without classic galactosemia. FSH bioactivity was measured in a subset of girls and women with and without galactosemia who were not on hormone therapy.

Results-FSH levels were significantly higher and AMH levels were significantly lower in our galactosemic cases relative to controls. FSH bioactivity did not significantly differ between cases and controls.

Conclusions-Close to $90 \%$ of girls and women with classic galactosemia have a profound absence of ovarian function, a deficit which is evident shortly after birth, if not before. These patients have no evidence of abnormally functioning FSH. AMH levels can be assessed prior to menarche or

(C) 2009 American Society for Reproductive Medicine. Published by Elsevier Inc. All rights reserved.

Corresponding author: Judith L. Fridovich-Keil, Department of Human Genetics, Emory University School of Medicine, Atlanta, GA, 30322; TEL: 404.727.3924; FAX: 404 727-3949; E-Mail: jfridov@emory.edu.

Authors contributed equally to this work.

Publisher's Disclaimer: This is a PDF file of an unedited manuscript that has been accepted for publication. As a service to our customers we are providing this early version of the manuscript. The manuscript will undergo copyediting, typesetting, and review of the resulting proof before it is published in its final citable form. Please note that during the production process errors may be discovered which could affect the content, and all legal disclaimers that apply to the journal pertain.

Capsule: Ovarian insufficiency in classic galactosemia is demonstrated by decreased AMH levels and is evident by early childhood; the cause appears to be direct ovarian toxicity rather than abnormal FSH function. 
after initiation of hormone therapy and may supplement FSH as a useful blood biomarker of ovarian function for patients with classic galactosemia.

\section{Keywords}

galactosemia; ovarian insufficiency; AMH; FSH; FSH bioactivity

\section{INTRODUCTION}

Classic galactosemia is an autosomal recessive disorder which results from profound impairment of galactose-1-phosphate uridylyltransferase (GALT) (reviewed in (1)), the second enzyme in the Leloir pathway of galactose metabolism (Figure 1). Classic galactosemia affects $\sim 1: 47,000$ newborns (2). In the first weeks of life, untreated infants develop vomiting, jaundice, hepatosplenomegaly and $E$. coli sepsis. These life-threatening symptoms can be reversed or prevented by dietary restriction of galactose (reviewed in (1)). However, despite neonatal diagnosis and careful dietary management, many patients experience serious long-term complications which can include cognitive, motor, and speech delays. Further, $>80 \%$ of girls and women with classic galactosemia experience premature ovarian insufficiency (POI (3)) which can range in severity from primary complete gonadal failure without the development of secondary sex characteristics to premature menopause (4-6). Girls with this disorder may require hormone therapy to complete puberty, and quality-of-life studies indicate that infertility is a significant cause of concern for galactosemic girls and their parents (7).

The developmental timing and underlying cause of galactosemia-associated POI remain unknown. The presumed etiology involves abnormal accumulation of galactose metabolites both pre- and postnatally, although the target and pathway of toxicity remain unclear (reviewed in (1)). Anecdotal studies of patients have offered some insight, although the small numbers of patients studied and the recognized variability of outcome among different patients confound sweeping conclusions. For example, Levy and colleagues (8) reported finding apparently normal folliculogenesis and abundant oocytes in an ovary from a 5 day old infant who died from complications of galactosemia. In contrast, Kaufman and colleagues (9) reported patients between the ages of 9-29 years who demonstrated decreased or completely absent ovarian tissue with pelvic ultrasonography. Similarly, Fraser and colleagues (10) reported that ovarian biopsies from two sisters with galactosemia demonstrated true ovarian failure in one sister and resistant ovary syndrome, defined by the presence of unresponsive primordial follicles, in the other. These authors hypothesized that resistant ovary syndrome might precede true ovarian failure in patients with galactosemia. Another ostensible case of resistant ovary syndrome was reported by Twigg and colleagues (11), who described a patient treated with hormone replacement early in puberty; this young woman later experienced a series of menstrual cycles even after hormone replacement was terminated.

Studies in animal models also support a link between galactose metabolism and ovarian toxicity. For example, adult female rats fed a high galactose diet have diminished follicular development (12), and galactose exposure in utero both inhibits germ cell migration to the developing ovary (13) and reduces oocyte pools (14). In contrast, a GALT knock-out mouse (15) has no apparent reproductive compromise, even when challenged with a high galactose diet (16). The basis for this apparent galactose-sensitivity of human and rat ovaries, in contrast to the apparent galactose-insensitivity of mouse ovaries, remains unclear.

Aside from its role in energy metabolism, the Leloir pathway is also responsible for regulating the supply of UDP sugars necessary for post-translational glycosylation of proteins and lipids; not surprisingly, patients with classic galactosemia exhibit glycosylation defects (reviewed in (1)). Glycosylation defects reported from studies of patient samples include reduced 
incorporation of galactosyl and galactosamine residues and loss of terminal galactose-linked sialic acid. These defects have been observed in a variety of sample types, including glycoproteins and glycolipids from post-mortem galactosemic brain tissue $(17,18)$, serum transferrin $(19,20)$, and the serum lysosomal enzymes $\beta$-hexosaminidase and $\alpha$-fucosidase (21).

Circulating follicle stimulating hormone (FSH) is also partially abnormally glycosylated in women with classic galactosemia (22). The glycosylation state of FSH is directly linked to its bioactivity in vivo, as well as to its rate of clearance, potency, and receptor binding and activation; hypoglycosylated isoforms of FSH may even act as antagonists (23). This observation raises the possibility that POI in women with classic galactosemia may arise from aberrant FSH function rather than from direct ovarian incompetence. In 2004, a case report described a woman with galactosemia-associated POI who successfully conceived after treatment with recombinant FSH (24). While intriguing, however, this result is difficult to generalize; recombinant FSH may have succeeded in this case by superovulating residual follicles rather than by replacing the patient's aberrant endogenous FSH.

Traditionally, studies of serum FSH levels have been used to predict ovarian insufficiency in galactosemic girls (reviewed in (1)), but this practice does not provide direct information about follicular function. Elevated FSH levels can indicate either depressed gonadal function or intrinsic hormone/receptor dysfunction. In addition, GnRH pulsatility may not yet be mature in pre-pubertal girls, further confounding interpretation of FSH levels.

In contrast, anti-Müllerian hormone (AMH), which plays an important role in folliculogenesis (25), is produced by the granulosa cells of preantral and small antral follicles of the ovary and levels are relatively stable across the menstrual cycle (26-28). AMH levels are also largely unaffected by oral contraceptives (29) or gonadotropin treatment (30), making AMH a particularly useful marker for patients on hormone therapy, as are many pubertal or postpubertal girls and women with classic galactosemia.

To distinguish between true gonadal insufficiency and aberrant FSH function in galactosemia we measured serum and/or plasma levels of AMH and FSH in 35 girls and women with classic galactosemia and in 43 age-matched controls; we also assessed FSH bioactivity in a subset of these study subjects. As predicted from the frequency of POI among galactosemic patients, only 2 of the 35 patients tested demonstrated normal levels of AMH; one additional patient demonstrated near-normal AMH. Further, while many patients demonstrated abnormally elevated levels of FSH, the bioactivity of that FSH remained normal. This result is consistent with an earlier report by Kaufman and colleagues (9) who applied an in vivo mouse method to test the function of urinary gonadotropins from 18 girls and women with galactosemia; no abnormality was detected in those samples. Combined, these results strongly favor the "direct ovarian toxicity" hypothesis of ovarian insufficiency in galactosemia and suggest that studies of AMH should supplement studies of FSH as measures of ovarian function in girls and women with classic galactosemia.

\section{MATERIALS AND METHODS}

\section{Subjects}

Girls and women between the ages of $<1$ to 46 years with classic galactosemia confirmed by biochemical and/or mutational analyses were enrolled in this study. Participation was solicited at the 2006 Parents of Galactosemic Children (PGC) Conference in Philadelphia, PA, by announcements posted online on the PGC website (www.galactosemia.org) and a discussion forum devoted to galactosemia (www.galactosemics.org), and through referral of patients from metabolic disorders clinics. 
Adult controls between the ages of 18 and 51 years who were not pregnant and had no selfreported history of impaired galactose metabolism, infertility, or neurological disorders were also enrolled in the study. Controls were solicited via announcements posted electronically and in hard copy on the Emory University (Atlanta, GA) campus. Anonymous plasma sample discards representing controls between the ages of $<1$ to 21 years were obtained from the Emory Genetics Laboratory (Atlanta, GA). This study was approved by the Institutional Review Board of Emory University. With the exception of anonymous controls, informed consent was obtained from all subjects or their legal guardians. None of the authors has a conflict of interest regarding this work.

Assays

Sample Collection/Storage-All blood samples were drawn without regard to menstrual cycle day. Blood draws on patients were performed either at the 2006 Parents of Galactosemic Children, Inc. (PGC) conference or in conjunction with routine clinical venipuncture. All blood samples were collected and stored overnight (or shipped overnight), then aliquotted and frozen. Using a small number of control bloods we compared AMH and FSH levels in samples frozen immediately or after overnight storage; storage for $\leq 24$ hours had no effect on AMH or FSH values.

For measurements from serum, $\sim 5 \mathrm{~mL}$ of blood drawn into a serum gel separator tube was allowed to clot at room temperature (RT) and then centrifuged for 5 minutes at $1315 \mathrm{rcf}$ in a fixed-angle rotor centrifuge. Each serum sample was transferred to a fresh tube, stored for no more than 24 hours at $0-4^{\circ} \mathrm{C}$, and then aliquotted to smaller volumes and stored at $-85^{\circ} \mathrm{C}$ until use.

For measurements from plasma, $\sim 5 \mathrm{~mL}$ of blood drawn into a sodium heparin tube was allowed to sit at RT or $0-4^{\circ} \mathrm{C}$ for no more than 24 hours and then centrifuged at $1800 \mathrm{rpm}$ for 15 minutes in a swinging bucket rotor; plasma was collected and aliquots were stored at $-85^{\circ} \mathrm{C}$ until use. Anonymous pediatric control plasma samples were obtained pre-frozen and stored at $-85^{\circ} \mathrm{C}$ until use.

AMH measurements-AMH levels in plasma or serum were determined using the AMH/ MIS ELISA kit from Diagnostic Systems Laboratories (cat.\# DSL-10-14400). Samples were assayed in duplicate following the manufacturer's instructions. For many samples both serum and plasma were available; independent assays of both sample types from the same study subject demonstrated indistinguishable results. Assays were performed by the Biomarkers Core Facility of Yerkes Primate Research Center at Emory University (Atlanta GA), or by the Reproductive Endocrine Unit Laboratory, Massachusetts General Hospital (Boston MA). Duplicate samples sent to both laboratories yielded indistinguishable results. Lower limit of detection for this assay was $0.01 \mathrm{ng} / \mathrm{mL}$, and inter-assay CV value was $11.42 \%$.

FSH measurements-Levels of FSH in plasma or sera were quantified by immunoradiometric assay using a kit from Diagnostic Systems Laboratories (cat.\# DSL-4700). Samples were assayed in duplicate according to the manufacturer's protocol. Assays were performed by the Biomarkers Core Facility of Yerkes Primate Research Center at Emory University (Atlanta GA). As for AMH studies, comparisons of FSH results from plasma and sera from the same study subjects yielded indistinguishable results. Lower limit of detection for this assay was $1.50 \mathrm{mIU} / \mathrm{mL}$; inter-assay and intra-assay $\mathrm{CV}$ values were $3.56 \%$ and $7.71 \%$, respectively.

For both AMH and FSH measurements, samples were batched to minimize assay-to-assay variation. Each batch contained both control and galactosemic samples. In all cases, persons 
performing the assays were blinded as to whether specific samples represented patients or controls.

FSH bioactivity-Bioactivity measurements were performed on samples collected from a subset of subjects $\geq 8$ years of age who were receiving no exogenous hormones. Bioactivity of FSH in plasma samples was determined using Chinese Hamster Ovary (CHO) cells transfected to express the human FSH receptor (FSHR) (manuscript in preparation). Briefly, the assay is a modification of other transfected cell assays in which cells produce cAMP in response to receptor activation by FSH (31-34). CHO-FSHR cells were incubated with patient samples at a final concentration of $12.5 \%$ plasma with added phosphodiesterase inhibitor (IBMX). cAMP production was quantified by a commercial RIA kit (Perkin-Elmer Life Sciences, Boston MA) and compared to a cAMP standard curve generated by recombinant $\mathrm{hFSH}$ at doses of 0.18 to $56 \mathrm{mIU} / \mathrm{ml}$. Total bioactivity of a given sample was then divided by total quantity of FSH in that sample to obtain the B/I ratio, allowing for bioactivity comparisons between samples containing different amounts of FSH.

\section{Statistical Analyses}

Analyses of AMH and FSH Levels-We applied regression procedures to assess the relationship between galactosemia and hormone levels of interest. To circumvent the nonnormality of the distributions of both AMH and FSH, we created dichotomized forms of these two outcomes. Specifically, we developed a binary outcome of abnormal AMH (1 if AMH < 1.5, 0 otherwise) and also created a binary outcome of abnormal FSH ( 1 if FSH > 10, 0 otherwise). We then applied logistic-regression procedures that regressed each of these binary outcomes on galactosemia status ( 1 if the subject is galactosemic, 0 if the subject is a control), adjusting for the effects of age. Given the apparent non-linear relationship between age and hormone levels, we modeled the former as a four-level categorical covariate (with each level containing an equal number of subjects) and treated the youngest level as the baseline category. To ensure that our results were robust to small-cell counts in the data, we avoided the use of asymptotic theory for significance and instead based significance on 10,000 random permutations of the data. We also used exact procedures to calculate odds ratios (OR) and 95\% confidence intervals (CI). We conducted these analyses using appropriate components of the $S A S v .9 .1$ and $R$ software packages.

Analysis of FSH Bioactivity-For FSH bioactivity, we calculated mean B/I ratios for both patient and control groups. We then compared the means of the two groups using a two-tailed Student's T-test assuming equal variances of the groups; this assumption was supported by an $\mathrm{F}$ test of variance equality. A p-value of $<0.05$ was considered statistically significant. We conducted these analyses using appropriate components of the Microsoft Excel software package.

\section{RESULTS}

\section{Study Volunteers}

We enrolled a total of 35 girls and women with classic galactosemia ranging in age from 10 months to 46 years, and 43 control girls and women ranging in age from 6 weeks to 51 years (Table 1). Mean age of the galactosemic group was 14.3 years; mean age of the control group was 24 years. The majority of enrolled subjects in both groups were $\geq 8$ years old; in this subset we identified 10 patients and 8 controls who were not taking oral contraceptives or receiving hormone replacement therapy. For all parameters measured, the values detected in our control group corresponded to previously published normal ranges $(27,28,35)$. 


\section{AMH levels in most galactosemic girls and women are abnormally low}

We measured serum or plasma AMH as an indicator of functional follicular reserve in girls and women with classic galactosemia. Levels obtained for 33 patients and 13 controls were below the assigned cutoff value of $>1.50 \mathrm{ng} / \mathrm{mL}$ for normal AMH (Figure 2, Table 2); this is a conservative cut-off based on previously reported ranges seen in girls and reproductive-age women $(27,28,35)$. Across all age groups, AMH values in our patients were significantly lower than in our controls (Table 2). Age-adjusted logistical regression analysis for AMH value below $1.5 \mathrm{ng} / \mathrm{mL}$ yielded an exact odds ratio of 104.22 (95\% CI 12.48 to $>1000$, p-value <0.0001) for patients as compared to controls.

\section{FSH levels in many galactosemic girls and women are high}

We also evaluated gonadal function indirectly by measuring circulating FSH levels in our subjects. 16 patients and 4 controls had FSH values above the assigned cutoff of $10 \mathrm{mIU} / \mathrm{mL}$ for normal FSH (Figure 3, Table 2); all controls with FSH $>10 \mathrm{mIU} / \mathrm{mL}$ were either $<2$ yrs or $>40$ yrs old, ages at which FSH is either unreliable or expected to rise. Using logistical regression analysis and adjusting for age, we confirmed the expected outcome - that having galactosemia was significantly associated with abnormally high FSH levels. The exact odds ratio for high FSH $(>10 \mathrm{mIU} / \mathrm{mL})$ in patients relative to controls was 10.58 with a 95\% confidence estimate of 2.36-68.51 (p-value $=0.0006$ ). As expected, the two galactosemic girls with normal AMH values (Figure 2) also demonstrated normal FSH levels (small arrows, Figure 3). Of note, despite the elevated FSH level of galactosemic patients as a group, more than half of all patients individually demonstrated normal FSH levels (Figure 3, Table 2).

\section{FSH bioactivity in galactosemic girls and women is normal}

To determine whether the FSH produced by galactosemic girls and women is functional, we assessed FSH bioactivity in a subset of subjects $\geq 8$ years of age who were not on oral contraceptives or hormone replacement (Figure 4, Table 2). The mean B/I ratio for the galactosemic group was 2.09 (range, 1.44-4.43) compared to a mean B/I ratio of 1.59 (range, 1.00-2.94) for controls. The difference in mean $B / I$ ratio between the two groups was not statistically significant $(\mathrm{p}=0.221)$.

\section{DISCUSSION}

The purpose of this study was to utilize serum or plasma biomarkers to explore the basis of hypergonadotrophic hypogonadism in girls and women with classic galactosemia. Specifically, we compared the predictions of two hypotheses that suggested either (1) that most galactosemic girls and women have diminished follicular function, or (2) that most galactosemic girls and women have functionally abnormal FSH, leading to secondary or apparent gonadal failure $(4,5,9,10,22,36,37)$. Our data overwhelmingly support the first hypothesis, and contradict the second.

Across age groups, the vast majority of our female galactosemic subjects demonstrated extraordinarily low AMH levels relative to age-matched controls, suggesting that follicular dysfunction occurs unusually early in life, ostensibly prior to menarche, and perhaps even prior to birth. Of note, there was no clear relationship between AMH value and GALT genotype in our cohort, although we did not have any patients in this study who carried mutations that have been recognized previously as mild (e.g. S135L, reviewed in (1)). Of further note, Knauff and colleagues (38) recently reported that serum AMH levels in galactosemia carriers are normal, consistent with the clinical reality that heterozygotes show no evidence of ovarian insufficiency. 
The percentage of galactosemic girls and women demonstrating abnormally low AMH levels in our study was $>90 \%$-- a number in striking agreement with the percentage of girls and women with classic galactosemia who demonstrate clinical evidence of POI (>80\%) (4-6). Of note, both of the patients in our study who demonstrated normal AMH levels are pre-menarche (at ages 2 yrs and $13 \mathrm{yrs}$ ), as is a third patient (10 yrs) whose AMH level $(1.45 \mathrm{ng} / \mathrm{mL})$ just missed the normal cut-off $(1.5 \mathrm{ng} / \mathrm{mL})$. It will be particular interesting to track the AMH levels and clinical progression of these 3 girls over time to see if their AMH levels remain within or near the normal range, and also to see whether these girls grow and mature to experience normal puberty and regular menses.

Our data also demonstrated that while the FSH level in our cohort of galactosemic girls and women, as a group, was significantly elevated, this marker was not elevated in all patients, especially in the pre-pubertal state. For example, of 11 galactosemic girls ages $<8$ yrs, 6 demonstrated normal FSH, yet of these 6 only 1 demonstrated normal AMH. Were a normal FSH level a reliable indicator of ovarian sufficiency in galactosemic girls, these data would predict a prevalence of POI in galactosemia of $<50 \%$, a value clearly at odds with the clinical data. Further, bioactivity measurements of FSH in our subjects with galactosemia were statistically indistinguishable from those of age-matched controls, thereby contradicting the hypothesis that galactosemic girls and women produce "functionally abnormal" FSH. Of course, our limited data set cannot rule out the possibility that studies of larger numbers of samples might identify a subtle difference in FSH bioactivity not detected here. However, given that 8 of the 10 patients studied here demonstrated FSH bioactivities well within the control range, our data suggest that any potential small difference in FSH bioactivity in some patients would be unlikely to account for the ovarian insufficiency seen in $>80 \%$ of girls and women with galactosemia. Of course, it is also possible that there may be additional FSH function(s) or receptors in humans other than the one represented by the assay we used here; we may therefore have missed some difference between cases and controls with regard to that function.

Our findings regarding AMH and FSH do not support previous hypotheses of a resistant ovary syndrome in galactosemia $(10,37)$; in contrast, it appears that the decreased follicular function or reserve is present from a very young age, at least in some patients. The cause for the variability in ovarian function among galactosemic women is not clear. Previous authors have proposed that higher amounts of galactose in the diet may positively correlate with FSH levels in metabolically normal women of late reproductive age (39). However, to what extent rigorous adherence to a galactose-restricted diet might delay follicular atresia in girls with galactosemia is uncertain. Studies in rats have shown that a high galactose diet, but not a high lactose diet, reduces the follicular count in subsequently harvested ovaries, possibly via down regulation of GDF-9 on the developing follicle $(40,41)$. Given the abnormal AMH levels in even our youngest galactosemic subjects $(<1$ year) it seems likely that ovarian insufficiency in patients may be evident at birth, suggesting that the ootoxic effects of gal-1-P or other galactose metabolites likely begin in utero. This hypothesis is supported by evidence from pregnant rats fed a high concentration of galactose, who subsequently gave birth to female pups with significantly decreased numbers of oocytes (14).

Our study represents a first step toward understanding the basis of POI in girls and women with classic galactosemia. In the near future we plan to increase the size of our subject and control populations. In addition, we will implement longitudinal studies to follow the progression of $\mathrm{AMH}, \mathrm{FSH}$, and clinical outcomes for galactosemic girls ascertained at a young age, which will be especially important for the few girls who demonstrate normal AMH levels in childhood. We also plan to conduct genetic and biochemical studies to investigate why some girls with classic galactosemia demonstrate normal AMH levels-and presumably preserved ovarian function-while most do not. 
Our studies to elucidate the pathophysiology of impaired ovarian function in galactosemia may ultimately provide insight into other mechanisms of POI that do not involve galactosemia. Association with POF or POI has been described in autoimmunity, disruption of germ-cell migration, other genetic disorders, Turner's syndrome, and possibly environmental or infectious etiologies. Some of these causes may share an as yet uncharacterized common pathway with POI in classic galactosemia. One recently proposed mechanism is via gal-1-P activation of the short form of the prolactin receptor in the ovary, which leads to premature follicular depletion in mice (42). Combined, these patient and animal studies may provide greater insight into normal processes of ovarian development and function, as well as causes of ovarian dysfunction in galactosemia and other conditions.

\section{ACKNOWLEDGMENTS}

We are especially grateful to the leadership and families of Parents of Galactosemic Children, Inc. and to the many study volunteers without whose participation this project would have been impossible. We also thank Dr. Rani Singh, Ms. Mary Jane Kennedy, Dr. Chunli Yu, and Dr. Stephanie Sherman for their assistance in obtaining study samples, and Dr. Patrick Sluss, Ms. Susie Lackey, and Ms. Ilana Brown for their assistance with the AMH and/or FSH studies. We further thank Dr. Ichiro Matsumura for helpful discussions early in this project

Financial Support: This work was supported by National Institutes of Health research grants R01DK059904 (to JLFK) and RO1DK063224 (to JWL). RDS was supported in part by training grant 2T32GM008367-16 from the National Institutes of Health (B. Shur and K. Wilkinson, co-PIs).

\section{REFERENCES}

1. Fridovich-Keil, J.; Galactosemia, WalterJ. Online Metabolic and Molecular Bases of Inherited Disease — OMMBID. Valle, D., et al., editors. Vol. Chap. 72. New York: McGraw-Hill; 2008. www.ommbid.com

2. Suzuki MWC, Beutler E. Large-scale molecular screening for galactosemia alleles in a pan-ethnic population. Hum Genet 2001;109:210-215. [PubMed: 11511927]

3. Welt C. Primary ovarian insufficiency: a more accurate term for premature ovarian failure. Clin Endocrinol (Oxf). 2008In press

4. Waggoner DD, Buist NRM, Donnell GN. Long-term Prognosis in Galactosemia: Results of a Survey of 350 Cases. J. Inher. Metab. Dis 1990;13:802-818. [PubMed: 1706789]

5. Guerrero NV, Singh RH, Manatunga A, Berry GT, Steiner RD, Elsas LJ. Risk factors for premature ovarian failure in females with galactosemia. J Pediatrics 2000;137:833-841.

6. Forges T, Monnier-Barbarino P, Leheup B, Jouvet P. Pathophysiology of impaired ovarian function in galactosaemia. Hum Reprod Update 2006;12:573-584. [PubMed: 16835432]

7. Bosch AM, Grootenhuis MA, Bakker HD, Heijmans HSA, Wijburg FA, Last BF. Living With Classical Galactosemia: Health-Related Quality of Life Consequences. Pediatrics 2004;113:e423-e428. [PubMed: 15121984]

8. Levy H, Driscoll S, Porensky R, Wender D. Ovarian Failure in Galactosemia. New England Journal of Medicine 1984;310:50. [PubMed: 6689742]

9. Kaufman FR, Kogut MD, Donnell GN, Goebelsmann U, March C, Koch R. Hypergonadotropic hypogonadism in female patients with galactosemia. N Engl J Med 1981;304:994-998. [PubMed: 6782485]

10. Fraser IS, Russell P, Greco S, Robertson DM. Resistant ovary syndrome and premature ovarian failure in young women with galactosaemia. Clinical Reproduction \& Fertility 1986;4:133-138. [PubMed: 3091236]

11. Twigg S, Wallman L, McElduff A. The resistant ovary syndrome in a patient with galactosemia: a clue to the natural history of ovarian failure. J Clin Endocrinol Metab 1996;81:1329-1331. [PubMed: 8636327]

12. Lai KW, Cheng LYL, Cheung ALM, O WS. Inhibitor of apoptosis proteins and ovarian dysfunction in galactosemic rats. Cell and Tissue Research 2003;311:417-425. [PubMed: 12658449] 
13. Bandyopadhyay S, Chakrabarti J, Banerjee S, Pal AK, Bhattacharyya D, Goswami SK, et al. Prenatal exposure to high galactose adversely affects initial gonadal pool of germ cells in rats*. Hum. Reprod 2003;18:276-282. [PubMed: 12571162]

14. Chen YT, Mattison DR, Feigenbaum L, Fukui H, Schulman JD. Reduction in oocyte number following prenatal exposure to a diet high in galactose. Science 1981;214:1145-1147. [PubMed: 7302587]

15. Leslie ND, Yager KL, McNamara PD, Segal S. A Mouse Model of Galactose-1-phosphate Uridyl Transferase Deficiency. Biochemical and Molecular Medicine 1996;59:7-12. [PubMed: 8902187]

16. Wehrli S, Reynolds R, Segal S. Evidence for function of UDP galactose pyrophosphorylase in mice with absent galactose-1-phosphate uridyltransferase. Molecular Genetics and Metabolism 2007;91:191-194. [PubMed: 17433749]

17. Haberland C, Perou M, Brunngraber EG, Hof H. The neuropathology of galactosemia: a histopathological and biochemical study. J. Neurophatol. Exp. Neurol 1971;30:431-447.

18. Petry K, Greinix HT, Nudelman E, Eisen H, Hakomori S, Levy HL, et al. Characterization of a novel biochemical abnormality in galactosemia: deficiency of glycolipids containing galactose or $\mathrm{N}$ acetylgalactosamine and accumulation of precursors in brain and lymphocytes. Biochem. Med and Metabolic Biol 1991;46:93-104.

19. Charlwood J, Clayton P, Keir G, Mian N, Winchester B. Defective galactosylation of serum transferrin in galactosemia. Glycobiology 1998;8:351-357. [PubMed: 9499382]

20. Sturiale L, Barone R, Fiumara A, Perez M, Zaffanello M, Sorge G, et al. Hypoglycosylation with increased fucosylation and branching of serum transferrin $\mathrm{N}$-glycans in untreated galactosemia. Glycobiology 2005;15:1268-1276. [PubMed: 16037488]

21. Jaeken J, Kint J, Spaapen L. Serum lysosomal enzyme abnormalities in galactosaemia. The Lancet 1992;340:1472-1473.

22. Prestoz L, Couto A, Shin Y, Petry K. Altered follicle stimulating hormone isoforms in female galactosaemia patients. Eur J Pediatr 1997;156:116-120. [PubMed: 9039515]

23. Barrios-De-Tomasi J, Timossi C, Merchant H, Quintanar A, Avalos JM, Andersen CY, et al. Assessment of the in vitro and in vivo biological activities of the human follicle-stimulating isohormones. Molecular \& Cellular Endocrinology 2002;186:189-198. [PubMed: 11900895]

24. Menezo Y, Lescaille M, Nicollet B, Servy E. Pregnancy and delivery after stimulation with rFSH of a galatosemia patient suffering hypergonadotropic hypogonadism: case report. J Assist Reprod Genet 2004;21:89-90. [PubMed: 15202737]

25. Visser J, Durlinger A, Peters I, van den Heuvel E, Rose U, Kramer P, et al. Increased oocyte degeneration and follicular atresia during the estrous cycle in anti-Müllerian hormone null mice. Endocrinology 2007;148:2301-2308. [PubMed: 17255205]

26. La Marca A, Stabile G, Carducci Artenisio A, Volpe A. Serum anti-Mullerian hormone throughout the human menstrual cycle. Human Reproduction 2006;21:3103-3107. [PubMed: 16923748]

27. Hehenkamp WJK, Looman CWN, Themmen APN, de Jong FH, te Velde ER, Broekmans FJM. AntiMullerian Hormone Levels in the Spontaneous Menstrual Cycle Do Not Show Substantial Fluctuation. J Clin Endocrinol Metab 2006;91:4057-4063. [PubMed: 16804046]

28. Tsepelidis S, Devreker F, Demeestere I, Flahaut A, Gervy C, Englert Y. Stable serum levels of antiMullerian hormone during the menstrual cycle: a prospective study in normo-ovulatory women. Hum. Reprod 2007;22:1837-1840. [PubMed: 17485437]

29. Somunkiran A, Yavuz T, Yucel O, Ozdemir I. Anti-Mullerian hormone levels during hormonal contraception in women with polycystic ovary syndrome. European Journal of Obstetrics \& Gynecology and Reproductive Biology 2007;134:196-201. [PubMed: 17335955]

30. Wachs DS, Coffler MS, Malcom PJ, Chang RJ. Serum Anti-Mullerian Hormone Concentrations Are Not Altered by Acute Administration of Follicle Stimulating Hormone in Polycystic Ovary Syndrome and Normal Women. J Clin Endocrinol Metab 2007;92:1871-1874. [PubMed: 17299061]

31. Tano M, Minegishi T, Nakamura K, Karino S, Ibuki Y. Application of Chinese hamster ovary cells transfected with the recombinant human follicle-stimulating hormone (FSH) receptor for measurement of serum FSH. Fertility \& Sterility 1995;64:1120-1124. [PubMed: 7589663] 
32. Albanese C, Christin-Maitre S, Sluss PM, Crowley WF, Jameson JL. Development of a bioassay for FSH using a recombinant human FSH receptor and a cAMP responsive luciferase reporter gene. Molecular \& Cellular Endocrinology 1994;101:211-219. [PubMed: 9397955]

33. Gudermann T, Brockmann H, Simoni M, Gromoll J, Nieschlag E. In vitro bioassay for human serum follicle-stimulating hormone (FSH) based on L cells transfected with recombinant rat FSH receptor: validation of a model system. Endocrinology 1994;135:2204-2213. [PubMed: 7956943]

34. Tilly JL, Aihara T, Nishimori K, Jia XC, Billig H, Kowalski KI, et al. Expression of recombinant human follicle-stimulating hormone receptor: species-specific ligand binding, signal transduction, and identification of multiple ovarian messenger ribonucleic acid transcripts. Endocrinology 1992;131:799-806. [PubMed: 1322283]

35. La Marca A, Stabile G, Artenisio AC, Volpe A. Serum anti-Mullerian hormone throughout the human menstrual cycle. Hum. Reprod 2006;21:3103-3107. [PubMed: 16923748]

36. Kaufman FR, Donnell GN, Roe TF, Kogut MD. Gonadal function in patients with galactosaemia. Journal of Inherited Metabolic Disease 1986;9:140-146. [PubMed: 3091920]

37. Russell P, Bannatyne P, Shearman RP, Fraser IS, Corbett P. Premature hypergonadotropic ovarian failure: clinicopathological study of 19 cases. International Journal of Gynecological Pathology 1982;1:185-201. [PubMed: 6820952]

38. Knauff E, Richardus R, Eijkemans M, Broekmans F, de Jong F, Fauser B, et al. Heterozygosity for the classical galactosemia mutation does not affect ovarian reserve and menopausal age. Reprod Sci 2007;14:780-785. [PubMed: 18089596]

39. Cooper GS, Hulka BS, Baird DD, Savitz DA, Hughes CL Jr, Weinberg CR, et al. Galactose consumption, metabolism, and follicle-stimulating hormone concentrations in women of late reproductive age. Fertility \& Sterility 1994;62:1168-1175. [PubMed: 7957979]

40. Liu G, Shi F, Blas-Machado U, Duong Q, Davis VL, Foster WG, et al. Ovarian effects of a high lactose diet in the female rat. Reproduction, Nutrition, Development 2005;45:185-192.

41. Liu G, Shi F, Blas-Machado U, Yu R, Davis VL, Foster WG, et al. Dietary galactose inhibits GDF-9 mediated follicular development in the rat ovary. Reproductive Toxicology 2006;21:26-33. [PubMed: 16105726]

42. Halperin J, Devi SY, Elizur S, Stocco C, Shehu A, Rebourcet D, et al. Prolactin Signaling Through the Short Form of Its Receptor Represses FOXO3 and its Target Gene Galt Causing a Severe Ovarian Defect. Mol Endocrinol. 2007me.2007-0399 


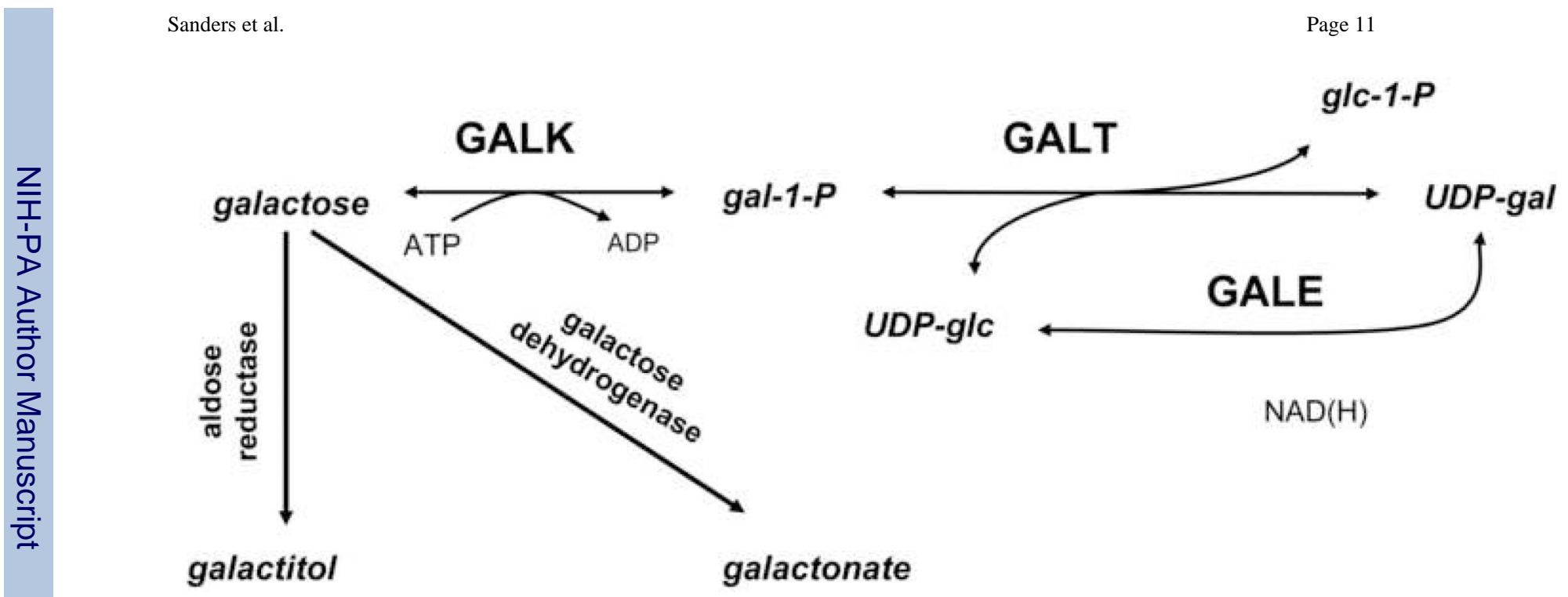

Figure 1.

The Leloir pathway of normal galactose metabolism. Classic galactosemia is caused by profound impairment of galactose-1-phosphate uridylyltransferase (GALT) leading to an increased accumulation of galactose, gal-1-P, galactitol and galactonate, and perhaps a depletion of UDP-gal and/or UDP-glc. 


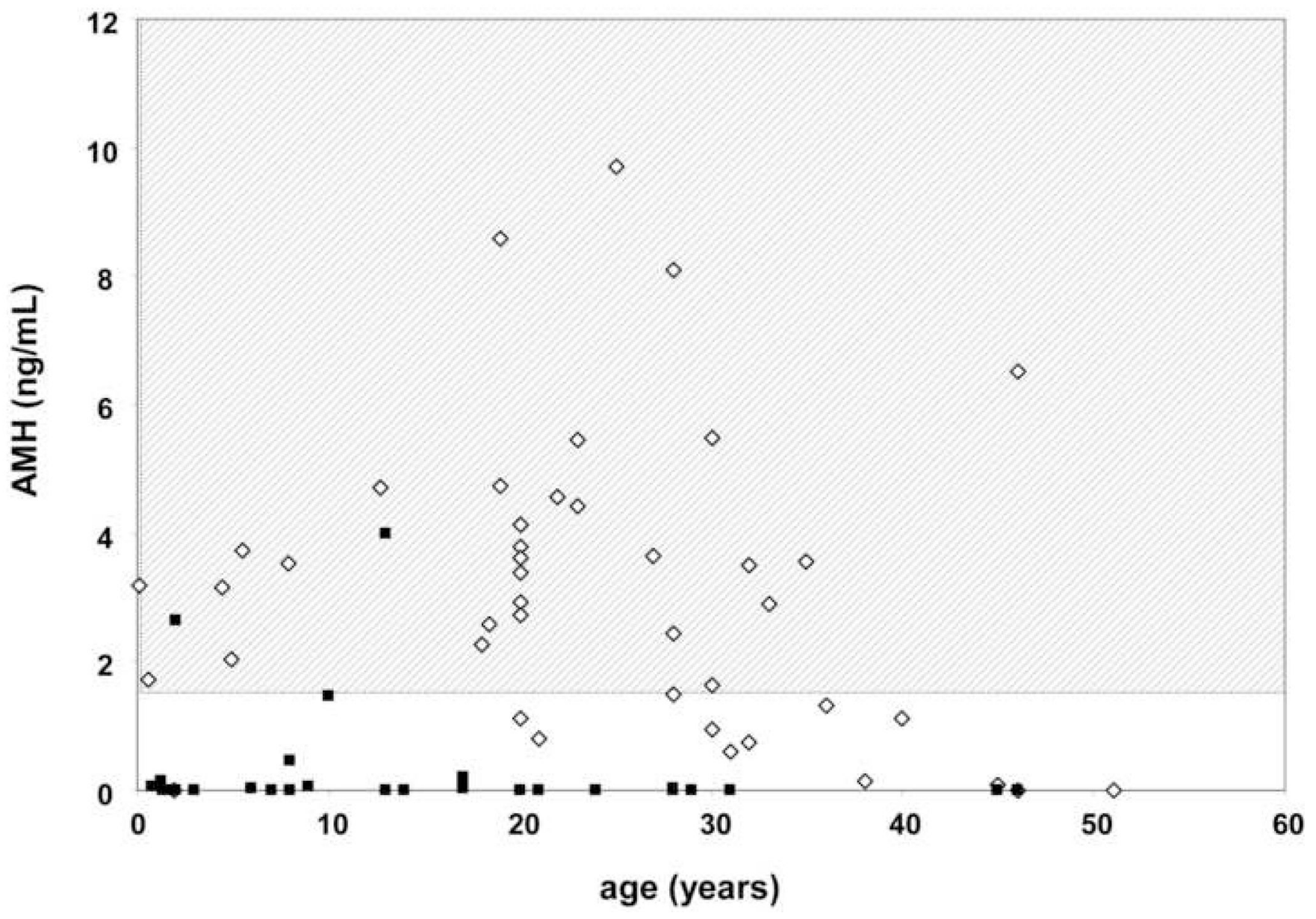

Figure 2.

AMH levels of girls and women with classic galactosemia (filled squares) and controls (open diamonds) between the ages of $<1$ and 52 yrs. Normal AMH range is shaded. 


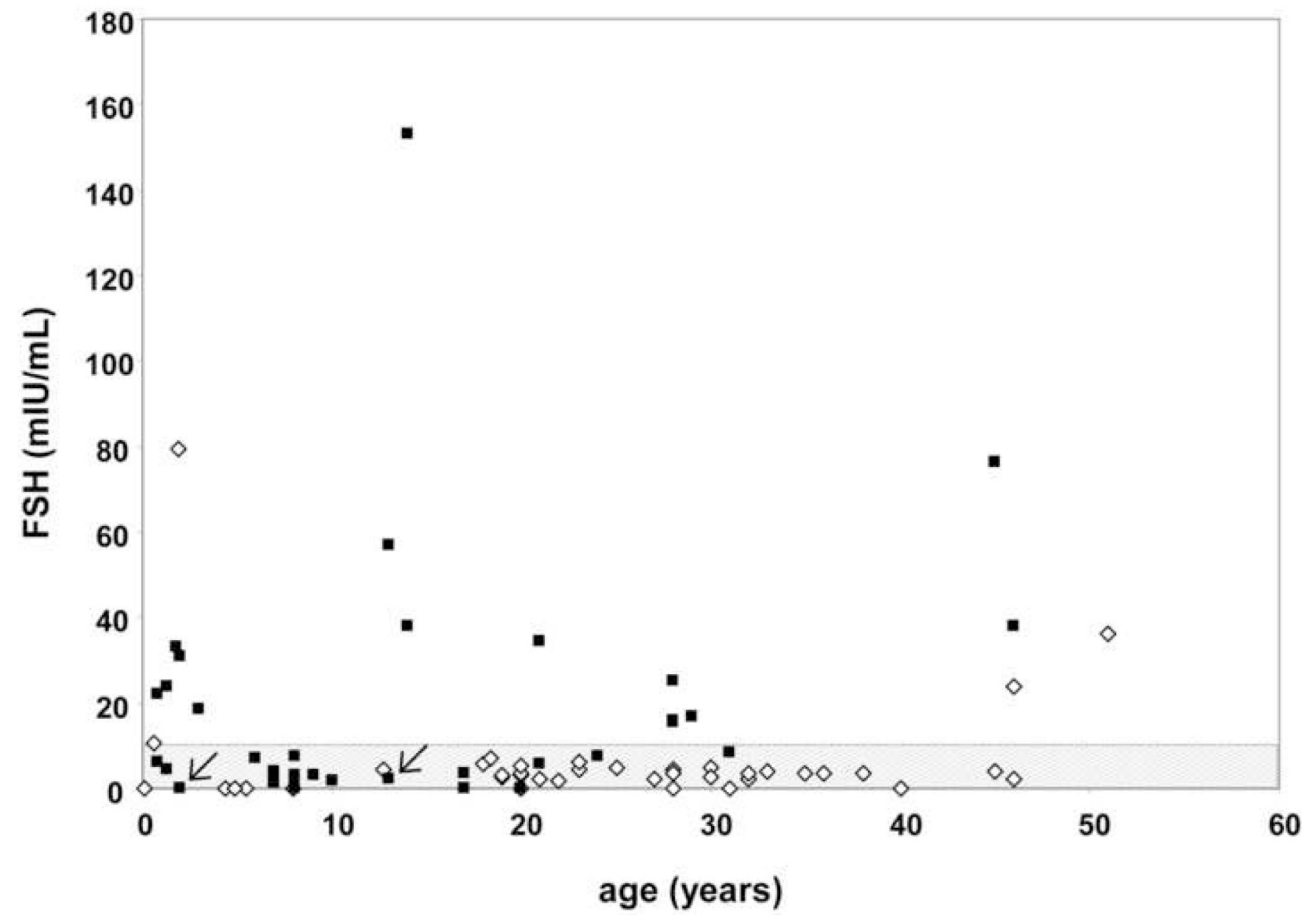

Figure 3.

FSH levels of girls and women with classic galactosemia (filled squares) and controls (open diamonds) between the ages of $<1$ and 51 yrs. Small arrows indicate the two patients in the study with normal AMH values (Figure 2). Normal FSH range in reproductive-age women is shaded. Note that FSH levels in very young girls ( $<2$ yrs) may vary, and women over 40 yrs are expected to demonstrate elevated FSH levels as they approach and enter menopause. 


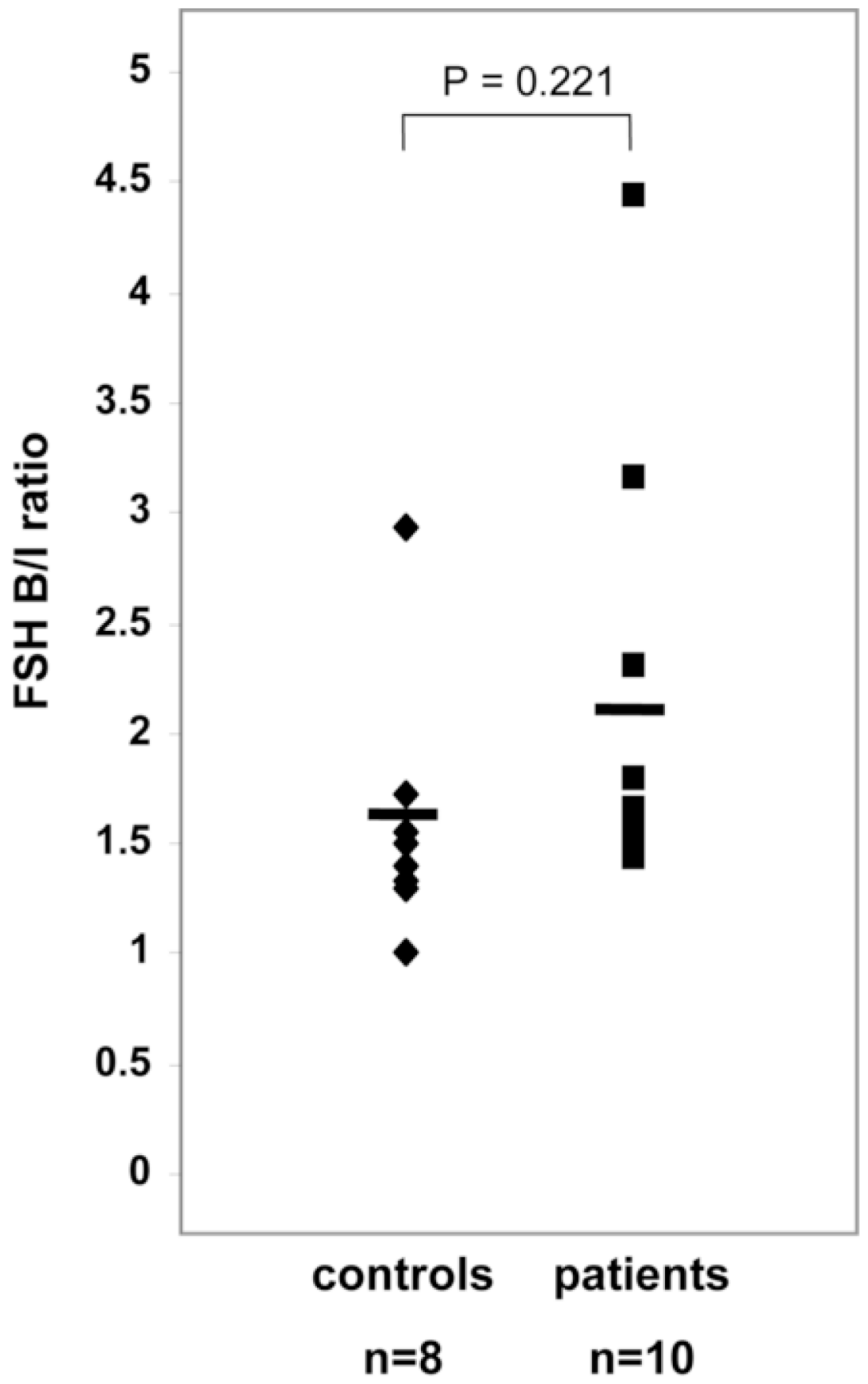

Figure 4.

FSH bioactivity represented by B/I ratio (activity divided by the circulating FSH level) in patients and controls $\geq 8$ yrs of age who were not on oral contraceptives or hormone therapy. Of note, 8 of 10 patients demonstrated FSH bioactivities that were within the control range; 2 presented FSH bioactivities that were slightly elevated. 
Subjects enrolled in the study

TABLE 1

The difference in mean ages between patients and controls was accommodated by regression analysis.

\begin{tabular}{|c|c|c|c|c|}
\hline Subjects Enrolled & & Controls & & Galactosemics \\
\hline & $\mathbf{n}$ & Mean age (range) & $\mathbf{n}$ & Mean age (range) \\
\hline Total subjects & 43 & $24.0(0.1-51)$ & 35 & $14.3(0.8-46)$ \\
\hline$<8$ yrs old & 7 & 3.6 & 11 & 3 \\
\hline$\geq 8$ yrs old & 36 & 28 & 24 & 19.5 \\
\hline not on OCPs or HRT & 8 & $35.8(20-51)$ & 10 & $14.3(8-29)$ \\
\hline
\end{tabular}




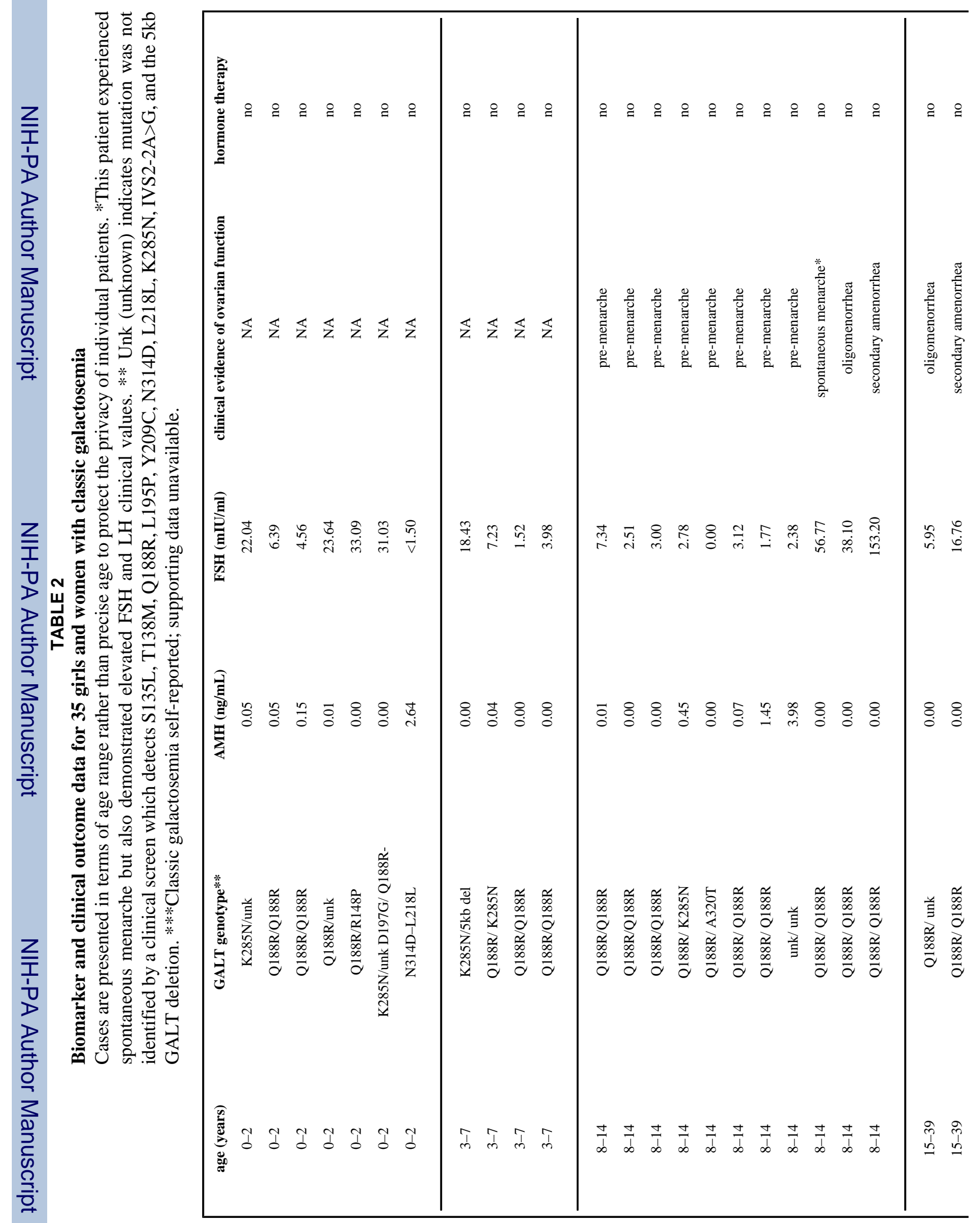

Fertil Steril. Author manuscript; available in PMC 2010 July 1. 


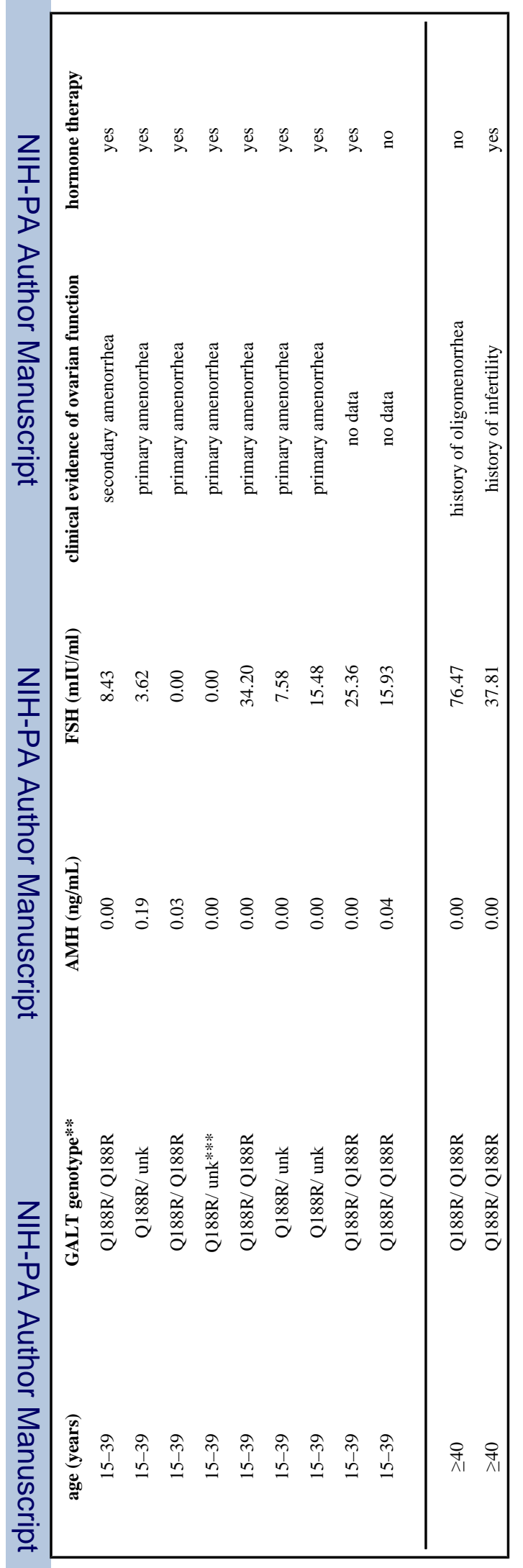

Fertil Steril. Author manuscript; available in PMC 2010 July 1. 